\title{
Optimization of leavening agents in extruded gluten-free brewer's rice hard pretzel using response surface methodology
}

\begin{abstract}
Celiac is the second most important issue in food sensitivities. The only treatment for celiac is a lifetime avoidance of consuming gluten. As pretzels are one of the most popular snacks in the world, making gluten-free pretzels is a market need. To produce gluten-free products, it is important to optimize the ingredients of the formulation. This research focused on optimization of the leavening agents of the most popular commercial hard pretzel formulation in which wheat flour is replaced with brewer's rice flour. This research also attempts to explain the effect of leavening agents in products containing no matrix molecules such as gluten. Response surface methodology via the central composite design was used to optimize the effects of yeast and bicarbonate of soda on the hardness (cutting strength), fracturability (brittleness), surface color, lateral expansion, expansion ratio, specific length, density (bulk, particle, material), porosity (open, close, total), water absorption index, water solubility index, water holding capacity, oil absorption index and oil holding capacity of gluten-free hard pretzel. The optimized results indicated that the gluten-free pretzel requires $8.05 \mathrm{~g}$ yeast and $2.59 \mathrm{~g}$ bicarbonate of soda for every $500 \mathrm{~g}$ of brewer's rice flour.
\end{abstract}

Keyword: Leavening agent; Gluten-free; Brewer's rice hard pretzel; Gluten-free pretzel; Brewer's rice; Response surface methodology 